\title{
Variaciones de los parámetros hematológicos, utilizando anticoagulantes EDTA K3 y EDTA K2
}

\author{
Mario Golac, Víctor Zevallos, Paul Chacón, María Muñoz, Roberto Rivas, Eduardo Verástegui
}

EAP de Tecnología Médica, Facultad de Medicina, UNMSM

Objetivos: Determinar variaciones de parámetros hematológicos, utilizando anticoagulantes K3 y K2 del EDTA.

Diseño: Observacional de corte transversal.

Institución: EAP de Tecnología Médica, Facultad de Medicina, UNMSM.

Participantes: 28 alumnos del último de año de la EAP de Economía de la Facultad de Ciencias Contables.

Intervenciones: Se tomó 56 muestras de sangre venosa, utilizando tubos al vacío con EDTA K3 y K2: Se hizo recuento de hematies, leucocitos, plaquetas, hemoglobina, hematocrito y formula leucocitaria. El muestreo fue por conveniencia y se procesó en el Servicio de Hematologia del Hospital Centro Médico Naval en dos tiempos diferentes: a las 2 y 24 horas de obtenida la muestra, conservadas a temperatura ambiente.

Principales medidas de resultados: Se utilizó la t de student y coeficiente de correlación de Pearson para muestras apareadas y el grado de asociación, respectivamente.

Resultados: A las dos primeras horas no se encontró diferencia estadísticamente significativa ( $p>0,025)$, a excepción de los basófilos. A las 24 horas, tampoco se observó variaciones significativas, con excepción de las plaquetas, observándose una diferencia en el tubo con EDTA k3, con $p<0,025$ y $r=0,48$.

Conclusiones: Ambas presentaciones pudieran ser usadas en el recuento hematológico; sin embargo, recomendamos el uso del EDTA k2, ya que presenta menores interferencias.

Palabras clave: Parámetros hematológicos, anticoagulante EDTA K2, anticoagulante EDTA K3.

\section{Alteraciones eritrocitarias más frecuentes en lámina periférica de pacientes con Enfermedad de Carrión}

Dante Acuña, Bruce Alvites, Hellen Baldeón, Oscar Cule, Luis Solano, Boris Valdivia, María Muñoz, Eduardo Verástegui

Instituto de Medicina Tropical Daniel A. Carrión y EAP de Tecnología Médica, Facultad de Medicina, UNMSM

Objetivos: Determinar cuáles son las alteraciones hematológicas más frecuentes en frotices de pacientes con la enfermedad de Carrión.

Diseño: Descriptivo, retrospectivo.

Institución: Instituto de Medicina Tropical y EAP de Tecnología Médica, Facultad de Medicina, UNMSM.

Material biológico: Frotices de sangre periférica de pacientes con diagnóstico de enfermedad de Carrión en fase aguda.

Intervenciones: Se estudió microscópicamente 50 muestras aptas de frotices de sangre periférica coloreadas con Giemsa, de pacientes con Enfermedad de Carrión, considerando como variables edad, lugar de procedencia, sexo y peso.

Principales medidas de resultados: Se estudió la frecuencia, análisis descriptivo, el OR para el análisis de variables cualitativas, promedio para variables cuantitativas, mediana, desviación estándar y coeficiente de variación para distribuciones asimétricas.

Resultados: Se encontró alteraciones a nivel de tamaño: 10 con microcitosis (20\%), 25 con anisocitosis ( $50 \%$ ) y $30 \%$ normal. En alteraciones en su forma: 5 muestras con dianocitos (10\%), 5 con acantocitos (10\%), 5 con estomatocitos (10\%), $70 \%$ normal. En alteraciones de color: 40 con hipocromía (80\%), 5 anisocromía (10\%); el 10\% restante fue considerada normal.

Conclusiones: Uno de los principales factores por las que no pudimos observar inclusiones eritrocitarias, fue que los extendidos y las coloraciones eran muy antiguas y habían perdido nitidez, color, mala conservación, humedad, entre otros factores. Sin embargo, en las láminas en las que se observó alteraciones propias de tal patología, fueron consideradas representativas para los resultados emitidos en el presente trabajo.

Palabras clave: Alteraciones hematológicas, Enfermedad de Carrión, frotis sanguíneo. 